\title{
A Comparison of Vocational Engagement among Young People with Psychosis, Depression and Borderline Personality Pathology
}

Caruana, E. ${ }^{1}$, Cotton, S.M. ${ }^{2,3}$, Farhall, J. ${ }^{1,4}$, Parrish, E.M. ${ }^{2,3,6}$, Chanen, A. ${ }^{2,3,4,5}$, Davey, C.G. ${ }^{2,3,5}$, Killackey, E. ${ }^{2,3}$ \& Allott, K.,

\section{Affiliations:}

${ }^{1}$ La Trobe University, Kingsbury Drive, Bundoora, Victoria, Australia, 3086.

${ }^{2}$ Orygen, The National Centre of Excellence in Youth Mental Health, 35 Poplar Road, Parkville, Victoria, Australia, 3052.

${ }^{3}$ Centre for Youth Mental Health, The University of Melbourne, Parkville, Victoria, Australia, 3052.

${ }^{4}$ North Western Mental Health, Victoria, Australia.

${ }^{5}$ Orygen Youth Health, 35 Poplar Road, Parkville, Victoria, Australia, 3052.

${ }^{6}$ Northeastern University, 360 Huntington Avenue, Boston MA, USA, 02115.

Author Emails (respectively): caruana.e@students.latrobe.edu.au, sue.cotton@ orygen.org.au, j.farhall@latrobe.edu.au, parrish.em@husky.neu.edu, andrew.chanen@orygen.org.au, c.davey@unimelb.edu.au, eoin.killackey@orygen.org.au, kelly.allott@orygen.org.au.

\section{Corresponding Author:}

Emma Caruana

Doctor of Clinical Psychology Candidate

La Trobe University

Bundoora Victoria Australia 3083

Tel: 0421428506

Email: caruana.e@students.latrobe.edu.au

\section{Funding:}

Specific funding provided for the development of this manuscript was obtained via EC's Doctor of Clinical Psychology budget from La Trobe University.

EC is supported by an Australian Postgraduate Award from La Trobe University. SC, EK and CGD are each supported by a Career Development Fellowship from the National Health and Medical Research Council (1061998, 1051891, and 1061757, respectively). EP was supported by a Presidential Global Scholarship from Northeastern University. AC is supported by the National Health and Medical Research Council. KA is supported by the Ronald Philip Griffiths Fellowship from the Faculty of Medicine Dentistry and Health Sciences, The University of Melbourne.

Conflict of interest statement: The authors declare that they have no conflict of interest. 


\begin{abstract}
Poor vocational engagement is well documented among young people experiencing first-episode psychosis (FEP). The aim of the present study was to establish and compare rates of vocational engagement across young people with first-episode psychosis, depression, and borderline personality pathology. A file audit was used to collect vocational data of young people aged 15-25 entering tertiary mental health treatment in 2011. Rates of vocational engagement were similar across groups, indicating that like those with FEP, young people with depression and borderline personality pathology experience impaired vocational engagement and are in need of targeted vocational interventions. Post hoc analysis indicated that that depression group had significantly more people who were partially vocationally engaged compared with the psychosis group, suggesting that vocational interventions might need to be targeted differently across different diagnostic groups. Future research should explore risk factors for vocational disengagement across diagnostic groups in order to inform intervention development.
\end{abstract}

Keywords: First episode psychosis; depression; borderline personality disorder; employment; education.

Abstract: 149 words

Total words: 4389 words 


\section{Introduction}

The twin frameworks of recovery orientation and early intervention guide mental health intervention policy in most high-income countries. A recovery orientation emphasises processes such as connectedness, hope and optimism about the future, identity, meaning in life, and empowerment (Leamy, Bird, Le Boutillier, Williams, \& Slade, 2011). Early intervention aims for early detection and treatment of mental illness in order to prevent illness progression to a more severe stage, including the prevention of functional impairment and societal disengagement (Jackson \& McGorry, 2009). With three-quarters of mental illnesses (including psychosis, depression, and borderline personality disorder) having their onset before the age of 24 (Chanen \& McCutcheon, 2013; Kessler et al., 2005), the main focus of early intervention has been upon youth (adolescents and emerging adults).

Vocational recovery is a vital consideration for recovery within a youth early intervention framework. Vocational recovery focuses on engagement in developmentally appropriate employment and/or enrolment in education. Engagement in vocational tasks can be conceptualised on a spectrum of varying quality of engagement that includes full engagement in education or work but with no noted difficulties, and partial engagement in education or work where an individual is engaged but with noted difficulties (e.g., poor attendance, performance, task completion). Governments and inter-governmental organisations have begun to refer to young people who are completely vocationally disengaged as "NEET', (Not in Employment, Education, or Training), which is an objective and ecologically valid measure of poor functioning (Organisation for Economic Co-operation and Development (OECD), 2012). This focus on vocational recovery prompted the International Association for Youth Mental Health to launch The International Declaration on Youth Mental Health (ACAMH Special Interest Group in Youth Mental Health) in 2013, recognizing the international importance of early intervention promoting vocational engagement, a key developmental task for this age group. This declaration provides guidelines for policy-makers on the minimum standards for mental health services for young people. It states that, by $2023,90 \%$ of young people will report being engaged in meaningful educational, vocational or social activity two years after first accessing specialist mental health support.

Youth is characterized by the normative developmental tasks of educational attainment and initial career-based employment (Killackey, Jackson, Gleeson, Hickie, \& McGorry, 2006). The onset of mental illness can disrupt normative vocational development, limiting future attainment of more skilled jobs, and lowering both employment status and income expectations (Waghorn \& Lloyd, 2005). For young people with first- 
episode psychosis (FEP), being competitively employed or in education during the early stages of treatment significantly predicts occupational recovery (participating in work or study) at 12-month (Major et al., 2010) and 5-year (Albert et al., 2011) follow-up, after treatment. These findings accord with common experience that, when applying for work, having appropriate educational attainment or previous work experience provides a competitive advantage.

Impaired vocational engagement is well documented in FEP, with approximately 40-50\% of young people unemployed at the time they first seek help (Fraser, Berger, Killackey, \& McGorry, 2006; Marwaha \& Johnson, 2004), and more than two-thirds not completing secondary school ( $M=21$ years, $S D=2$ years) (Killackey, Jackson, \& McGorry, 2008). This is considerably higher than population rate averages across 34 high-income countries, with 2015 data indicating that $13.9 \%$ of 15 - to 24 -year-olds were unemployed (Organisation for Economic Co-operation and Development (OECD), 2017), and 2009 data indicating that 18\% of 25- to 34-year-olds did not complete senior secondary school education (Organisation for Economic Cooperation and Development (OECD), 2011). The first five years following psychosis onset is the critical period when functional disability emerges (Birchwood \& Fiorillo, 2000), with a large component of this being due to vocational disengagement, making early intervention crucial.

Among youth, research on vocational engagement is less well established for diagnostic groups other than psychosis. Similar to psychosis, major depression and borderline personality disorder (BPD) are severe mental illnesses with a peak age of onset during adolescence and young adulthood (American Psychiatric Association, 2013; Andrews \& Wilkinson, 2002; Chanen \& McCutcheon, 2013; Kessler et al., 2005), creating potential disruption to normative vocational development. There has recently been increased interest in early intervention for depression (Allen, Hetrick, Simmons, \& Hickie, 2007; Merry, 2007) and BPD (Chanen \& McCutcheon, 2013), with longitudinal data showing that illness onset in adolescence is associated with increased risk of adverse developmental consequences in terms of subsequent psychopathology, neurobiological development, cognitive functioning, and emotional, social and occupational functioning (Allen et al., 2007; Chanen, Jovev, McCutcheon, Jackson, \& McGorry, 2008).

Most existing data on the vocational engagement of young people with depression comes from population surveys. Australian population-level research indicates that $13.68 \%$ of people aged 15 to 19 years and $48 \%$ of people aged 20 to 29 years of age with an affective disorder were not studying or working, compared with $6.84 \%$ and $13 \%$ of healthy aged-matched peers, respectively (Waghorn, Chant, Lloyd, \& Harris, 
2011). Similarly, in the USA, a prospective population study found those aged 18 to 30 years with depression had reduced participation in employment, reduced working hours, and earned less, compared with healthy young adults (Whooley et al., 2002). However, information is scant on the vocational engagement of patients receiving treatment for depression from mental health services - data that are necessary for rational planning of vocational services and supports. To the authors' knowledge, the only study of vocational engagement among young people (15-24 year olds) receiving treatment for depression reports that $29 \%(\mathrm{n}=125)$ of those attending one of two Australian primary care youth mental health services were "NEET" at some point (baseline, 12month follow-up, or both) (O’Dea et al., 2016).

We were able to identify only one study of vocational engagement among youth with BPD. This study found that among 15 to 25 year-olds with borderline personality pathology (3-9 DSM-IV BPD criteria; mean of 5.7 criteria), $33.3 \%$ were not studying or working upon entry to specialist clinical care (Sio, Chanen, Killackey, \& Gleeson, 2011).

Although vocational disengagement among young people with depression and BPD might be similar to that experienced by young people with FEP, further research is needed to inform the extent of the problem and vocational intervention development. It is expected that young people who seek and receive treatment, particularly at a tertiary mental health service, would be likely to experience even more severe symptoms, have poorer functioning, and experience a higher burden of disease, compared with population-level samples. Moreover, based on current literature and clinical experience, it is possible that youth with depression and BPD experience similar vocational impairment to those with FEP. Thus the aim of the present study was to establish rates of participation in employment and education upon entry into tertiary mental health care among young people with FEP, depression and BPD, and to compare these data among the three groups.

\section{Study Design}

\section{Method}

The study was a clinical audit of files from Orygen Youth Health (OYH), the State Government funded youth mental health service for 15-25 year-olds residing in western and north-western metropolitan Melbourne, Australia. Each file contains information compiled during the young person's treatment period, derived from various sources, and from high quality assessments conducted by trained clinicians. Data was collected from the period when the young person first entered the service. All files were assessed by the first author, a Clinical 
Psychology Doctoral candidate. The Melbourne Health Human Research Ethics Committee approved the project (QA2015097). There are no known conflicts of interest, and all authors certify responsibility.

\section{Participants}

The sample comprised young people aged 15 to 25 years who registered for the first time as a client of OYH in 2011. Participants attended one of three specialist prevention and early intervention programs: the Early Psychosis Prevention and Intervention Centre (EPPIC) for psychosis (McGorry, Edwards, Mihalopoulos, Harrigan, \& Jackson, 1996); the Youth Mood Clinic (YMC) for severe and complex depression (Rice et al., 2017); or the Helping Young People Early (HYPE) clinic for borderline personality pathology (subthreshold and full-syndrome BPD) (Chanen, McCutcheon, \& Kerr, 2014). Those attending EPPIC were experiencing a current psychotic episode. Those attending YMC had complex depression, operationalised as a score of 15 or more on the Patient Health Questionnaire-9 (Kroenke \& Spitzer, 2002) (indicating a moderate-severe current depressive episode), or non-psychotic bipolar disorder (usually in the depressed phase) (Rice et al., 2017). Those attending the HYPE clinic met criteria for 3 or more BPD criteria (American Psychiatric Association, 2013) on the Structured Clinical Interview for DSM-IV Axis II disorders (SCID-II) (First, Gibbon, Spitzer, Williams, \& Benjamin, 1997). In addition, all YMC and HYPE patients were required to have been assessed as being at moderate- to high-risk of harm to self or others or to have poor psychosocial functioning (e.g., limited social support network, poor independent living skills, homelessness, lack of meaningful time use, disengagement from education and/or employment) (Rice et al., 2017).

Participant diagnostic grouping for this study was based on the OYH intake criteria, with decisions made at service entry by an assessing clinician and multi-disciplinary clinical team, rather than an independent research assessment. EPPIC is mandated to accept all FEP cases in the geographically defined catchment area. YMC and HYPE have limited capacity, meaning that patients are prioritised on the basis of clinical severity. Files were excluded if one of the following criteria were met: (1) an individual was transferred between clinics during their treatment, so as to minimise inclusion of cases where diagnosis was unclear; or (2) the individual had previously been registered and treated at $\mathrm{OYH}$.

\section{Procedure}

Lists of clients first registered with the clinics in 2011 were accessed, in order to extract a random stratified sample. The EPPIC and YMC samples were stratified by age (15-18 years and 19-25 years), by gender because of potential differences between the cohorts (e.g., more males present with psychosis) and the EPPIC 
sample was stratified by each of its two geographical regions. Due to the smaller number of HYPE registrations in 2011, all files were audited, without stratification. The sample size for the EPPIC and YMC groups were matched to reflect the size of the BPD group for statistical analysis. If a file was audited but met exclusion criteria, resampling was performed if the valid sample was less than 50 and a replacement file was available (Figure 1). A greater number of files were randomized from YMC compared to EPPIC due to the project being scaled down in size during the file audit, and EPPIC being the final clinic to be randomized. YMC was audited before EPPIC, with initial target sample sizes of 64 per clinic. However due to the time constraints of the project, and HYPE only having 45 patients eligible for the study, it was decided to revise the target sample size to 50 to enable similar sample sizes across groups for statistical comparison, thus fewer files were randomized for EPPIC.

\section{(INSERT FIGURE 1)}

\section{Materials}

Following a pilot file audit, a file audit tool was developed to systematically assess clinical files to determine information reliably available and the face validity of this information. Primary sources of information were the statewide mental health registration form (MH1), the NorthWestern Mental Health Assessment form, and the Health of the Nation Outcome Scales (HONOS; completed for those 18 years and over) (Wing et al., 1998) and Health of the Nation Outcome Scales (Children and Adolescents; HONOSCA; completed for those under 18 years) (Gowers et al., 1999), which are routinely completed on entry to the service. Some variables were directly extracted from files, while others, detailed below, were coded based on qualitative information extracted from files, with a $10 \%$ subset of files being coded by a second rater to check for errors and to determine an estimate of inter-rater reliability. Using these sources, the file audit tool included the following measures.

Demographic characteristics of young people at entry into service: age, gender, country of birth, preferred language, English proficiency, whether an interpreter was required, Aboriginal or Torres Strait Islander person, government pension/benefit entitlement, marital status, living status, housing, route of entry into service, Mental Health Act legal status, and whether they were hospitalized at registration.

Education measures included highest level of education completed, extracted from the mental health registration form, and Item 13: Poor school attendance, extracted from the HONOSCA. In addition to these measures, qualitative information (such as schooling and past education, as well as current education and any 
descriptive information on these) was extracted from the assessment form. Two independent raters (EC, EP) coded individuals' current education status as: not studying; partial engagement in study (including poor attendance, a drop in grades or failing, and not engaging in or understanding study tasks); and studying.

Employment measures included Item 12: Problems with occupation and activities, extracted from the HONOS. Additionally, qualitative information (such as employment history and current employment, and any descriptive information on these) was extracted from the assessment form. Two independent raters (EC, EP) coded individuals' current employment status as: unemployed; partial engagement in employment (including poor attendance, poor performance and being on leave); and employed.

Vocational measures were derived from the qualitative information extracted from the assessment form. Where there was missing data for education or employment, vocational measures were coded based on available information. Quality of engagement (in employment, education or training) rated individuals as: NEET (not in employment, education or training); partially vocationally engaged (engaged in work, study, or both, but with noted difficulties, e.g., poor attendance, performance, task completion, etc.); and fully vocationally engaged (engaged in work, study, or both, with no difficulties noted). Type of vocational activity rated individuals as: NEET (not in employment, education or training); engaged only in education; engaged only in employment; and engaged in both education and employment.

\section{Statistical Analysis}

Statistical analysis was conducted using IBM $^{\circledR}$ SPSS $^{\circledR}$ Statistics Version 22. Means, percentages and frequencies of participant demographic characteristics on entry to OYH were established for each of the diagnostic groups, and group differences were assessed using anova or chi-squared analyses. Frequencies and percentages for education, employment and overall vocational engagement measures were also calculated. Chisquared analyses $\left(\chi^{2}\right)$ were used to compare frequencies of all vocational measures across groups. For any statistically significant $\left(\chi^{2}\right)$ analysis, follow-up post hoc tests of proportions were done with Bonferroni correction, to determine which of the three groups differed significantly. For the $10 \%$ of files that were coded by two independent raters, an inter-rater reliability Cohen's kappa ( $)$ statistic was calculated.

\section{Results}

\section{Sample Characteristics}


A total of 145 files were audited: 50 from mood and psychosis and 45 from the BPD group. Overall, the groups were similar on demographic characteristics (Table 1). Gender was a stratification variable for the mood and psychosis groups but not BPD. The BPD group had significantly more females, compared with psychosis and mood, and this reflects the clinical epidemiology of BPD (American Psychiatric Association, 2013). The psychosis group had significantly fewer people born in Australia (62.5\%) compared with BPD (95.5\%). The psychosis group also had significantly fewer young people entering their service on a voluntary basis $(66.7 \%)$, compared with mood $(97.7 \%)$ and BPD (100\%) groups. There was a significant difference across groups for hospitalization at service entry (yes/no) but this was no longer significant when Bonferroni correction was applied to post hoc analyses.

(INSERT TABLE 1)

\section{Inter-rater reliability for vocational variables}

Estimates obtained for inter-rater reliability of variables coded from qualitative information indicated 'almost perfect agreement' (Viera \& Garrett, 2005) for all variables, with Cohen's $\kappa=0.93, p<.001$ for current education status; $\kappa=1.00, p<.001$ for current employment status; and $\kappa=0.90, \mathrm{p}<.001$ for quality of engagement and $\kappa=1.00, p<.001$ type of engagement.

\section{Education}

Information on current education status was available for 131 files (Table 2). No significant differences were found between the mood, BPD and psychosis groups for current educational status, $\chi^{2}(4)=4.15$, $p=.386$, with almost half of the total sample not engaged in study at service entry. Of the 46 people partially engaged in study, $52.2 \%$ had poor attendance, $30.4 \%$ reported a drop in grades or were currently failing, and $17.4 \%$ were attending study but reported either not completing or not understanding the work. There were no significant differences between groups on highest education completed or HONOSCA Item 13: poor school attendance.

\section{(INSERT TABLE 2)}

\section{Employment}

Information on current employment status was available for 101 files (Table 3). There were no significant differences between the mood, BPD and psychosis groups on current employment status, $\chi^{2}(4)=2.62$, $p=.624$, with over half of the total sample unemployed at service entry. Of the 11 people partially engaged in 
employment, $63.7 \%$ were on leave due to their decision/circumstances, $9.1 \%$ were on leave due to their employer's request, and $27.3 \%$ reported having poor attendance. There were also no significant differences between groups on HONOS Item 12: problems with occupation and activities.

\section{(INSERT TABLE 3)}

\section{Overall Vocational Engagement}

Overall, vocational measures were based on the available information from 143 files (Table 4). For quality of engagement, there was a significant difference between groups, $\chi^{2}(4)=12.35, p=.015$ (Figure 2). Bonferroni corrected post hoc tests of proportions indicated the only difference between the three groups was with respect to partial engagement, with the mood group having significantly more people partially vocationally engaged, compared with the psychosis group. NEET levels were not significantly different across groups: $26.5 \%$ of the mood group; $24.4 \%$ of the BPD group; $46.9 \%$ of the psychosis group. There were no significant differences between mood, BPD and psychosis groups on type of vocational activity.

\section{(INSERT TABLE 4)}

\section{(INSERT FIGURE 2)}

\section{Discussion}

This novel study compared the extent of vocational engagement among young people with severe depression and severe borderline personality pathology with that of young people with psychosis, for whom there is established evidence of significantly impaired vocational engagement (Fraser et al., 2006; Killackey et al., 2008; Marwaha \& Johnson, 2004). The key finding is that rates of engagement in education and employment did not significantly differ among the three groups.

For young people with psychosis, $62.9 \%$ were unemployed, and $55.8 \%$ were not studying. This is a slightly higher rate of unemployment at the time of help-seeking, compared with previous studies that reported unemployment rates between 40\% - 50\% (Fraser et al., 2006; Marwaha \& Johnson, 2004). The educational disengagement rate was consistent with previous research, which has found that more than two-thirds of youth with psychosis do not complete secondary school (Killackey et al., 2008); however, it should be noted that educational attainment and status are different constructs. There were no statistically significant group differences between the psychosis group and other groups on both employment and education, with unemployment ranging from $46.9 \%$ to $62.9 \%$, and rates of not studying ranging from $41.5 \%$ to $55.8 \%$. These 
rates depict high level of disengagement among all patient groups and justifies the claim that mood disorders and BPD (including even subthreshold disorder) are as 'severe' as psychotic disorders in these domains.

When considering overall vocational engagement, NEET status was $26.5 \%$ for the severe depression group, $24.4 \%$ for the BPD group, and $46.9 \%$ for the psychosis group. Despite the psychosis NEET rate seeming higher than the other groups, and the a priori chi-square analysis being significant, this was not a significant difference when Bonferroni correction was applied to post hoc analyses. This may be an issue with statistical power. For young people with severe depression, this is a similar finding to a previous clinical study which found $29 \%$ of young people aged 15 to 24 years with major depression were NEET at some point during their episode of care through a primary care youth mental health service (O’Dea et al., 2016). This differs from previous population-level research that found that $13.68 \%$ of people aged 15 to 19 years with an affective disorder were not studying or working and $48.31 \%$ of people aged 20 to 29 years were not studying or working (Waghorn et al., 2011). The mood disorders group in the current study, with a mean age of 18.54 years, most closely compares with the 15 to 19 years group, which suggests that population-level data might underestimate the extent of impairment and need for vocational intervention among help-seeking individuals attending a specialist service by including lesser degrees of impairment. However, it is also important to note that age 20 to 29 population-level data indicated higher rates of disengagement compared with our group, which might reflect worsening of vocational impairment with the progression or persistence of illness, highlighting the need for early vocational intervention. Current BPD NEET rates are comparable with previous OYH data collected during 2005, which found $33.3 \%$ of young people aged 15 to 25 years with BPD were neither studying nor working at service entry (Sio et al., 2011). This suggests that, similar to young people with psychosis, young people with severe and complex depression and severe BPD experience high levels of impaired vocational engagement.

The current study also involved investigation of partial engagement in work and study. This refers to those who were engaged but had poor attendance or poor performance (a drop in task completion/grades or failing education). To the authors' knowledge, there is little research on vocational productivity outcomes, such as absenteeism and 'presenteeism' (physically present in the classroom or workplace; however, functionally absent (Cooper \& Lu, 2016)), among youth with mental illness. Such variables highlight the importance of the quality of vocational engagement, not just absolute rates, and are likely to add depth to analysis of service needs. When examining employment and education separately, groups did not significantly differ in their rates of partial engagement. However, when examining overall quality of engagement, combining both work and study, 
the findings indicated that the severe depression group had significantly more people partially engaged, compared with the psychosis group. This implies that for the severe depression group, there might be a greater need for programs to focus on maintaining vocational engagement or re-engaging in a pre-existing vocational activity, compared with the psychosis group where there may be more of a need to focus on entering into new or first-time vocational activities.

Of note, the psychosis group had significantly fewer people born in Australia, compared with the BPD group, and this might reflect migration as a contributing factor to the development of psychosis (Morgan, Charalambides, Hutchinson, \& Murray, 2010). The psychosis group also had significantly fewer people enter the service on a voluntary basis, compared with other groups, and this might reflect the effects of acute psychosis on judgment, safety, insight and capacity to consent to treatment.

An Australian policy report on mental health and work found that nearly half of people under 35 years on the Disability Support Pension had a psychological disability as their primary disability (Organisation for Economic Co-operation and Development (OECD), 2015). At present, vocational interventions, such as supported employment and education, are primarily designed for those experiencing psychosis (Killackey et al., 2006; Killackey et al., 2008). The authors are unaware of any vocational interventions developed or delivered routinely and specifically for young people with severe BPD or severe depression. One implication of the current findings is that early intervention in relation to vocation needs to be available to all diagnostic groups in order to truly assist those who can and want to work to be able to enter the workforce. It is currently unclear whether the vocational interventions developed for psychosis are applicable to BPD or mood disorders in young people. The current finding that the severe depression group had a significantly greater proportion who are partially vocationally engaged, compared with the psychosis group, tentatively suggests that the needs of each group might differ in important ways with regard to vocational supports. Future research exploring risk factors for vocational disengagement for each group would be beneficial, as these might be informative when developing interventions. Knowing whether predictors of disengagement differ across groups would also inform whether similar interventions can be used across groups or whether interventions will need to be tailored.

Strengths of the study include that it focused on patients receiving treatment from a tertiary mental health services rather than the general population, which means findings can inform treatment and service planning, and intervention development. The sample included young people across three clinical groups with similar levels of severity (due to service entry criteria), from the same geographical catchment area, and at the same point in time, which all enhance the validity of comparison. Furthermore, vocational engagement was 
measured as both rates of engagement and quality of engagement, and education and employment was considered both separately and combined. This allowed for a thorough exploration of vocational functioning, and expanded on existing data which to our knowledge is limited to absolute rates. Knowledge of quality of engagement is likely to add depth to analysis of service needs.

Limitations to the study include that the BPD group was not stratified by age or gender. Given a significantly greater proportion of females among the BPD group, compared with the other groups, gender might have been a confounding variable in this study, as Australian population data indicate women have higher educational attainment and lower rates of labour force participation than men (Workplace Gender Equality Agency, 2014). Furthermore, the sample in this study is derived from one mental health service catchment area, and might not be representative of the population of young people with severe mental disorders. However, this catchment covers more than $25 \%$ of Melbourne's population of four million and OYH is the only service for severe mental disorders in the region. It is also important to note that this is likely to be a functionally impaired sample due to mood and BPD clinics having poor psychosocial functioning as a potential intake criterion, and we acknowledge that findings may not generalize to other settings. Another limitation is the high proportion of missing data (employment: 44 out of 145 files; education: 14 out of 145 files), and that NEET was calculated only from available information. Missingness was associated with age (15-18 year olds more likely to be missing employment data; 19 - 25 year olds more likely to be missing education data), thus available data reflected the normative vocational task for the age of the client. This suggests that the most likely explanation of the missing data is that the clinician did not deem education or employment as relevant to record when it was not the developmentally normative task of the age group. Thus, although our data may have missed some instances of either educational or employment engagement; we calculated NEET based on the available information as this was deemed to be the best estimate of vocation. Other limitations are that the diagnostic grouping was based on the OYH clinic assignment at service entry and on the basis of an initial clinical diagnosis, rather than a standardized research diagnosis. Also, co-morbidity is common among young people attending OYH (and youth mental health services generally), which means that the diagnostic groupings were not 'pure'. Using clinic assignment as a proxy for diagnosis might not be reliable, and it is possible that, for example, someone attending the early psychosis clinic might also have major depression. Strategies were employed to minimise the impact of this, such as excluding files where the young person had transferred between clinics, in order to ensure that those files included had a clear primary presenting problem that remained consistent across the episode of care with the service. While acknowledging this as a limitation, at a 
pragmatic level, when using the current findings to inform the need for vocational intervention, it is likely that co-morbidity and initial diagnostic uncertainty will be common occurrences within tertiary youth mental health services. This study is also limited because the possible effects of comorbid disorders (including intellectual, psychiatric and physical disorders) on vocational disengagement was not measured.

Overall, the findings from this study indicate that high rates of vocational disengagement are not limited to young people with psychotic disorders. Young people presenting to specialist mental health services for other mental health disorders that have their onset during adolescence and young adulthood, including severe and complex major depressive episodes and severe borderline personality pathology also have high rates of vocational disengagement, and are in need of vocational intervention.

Ethical approval: All procedures performed in studies involving human participants were in accordance with the ethical standards of the institutional and/or national research committee and with the 1964 Helsinki declaration and its later amendments or comparable ethical standards. For this type of study formal consent is not required.

\section{References}

ACAMH Special Interest Group in Youth Mental Health. (2013). The International Declaration on Youth Mental Health Retrieved from http://www.iaymh.org/f.ashx/8909 Int-DeclarationYMH print.pdf

Albert, N., Bertelsen, M., Thorup, A., Petersen, L., Jeppesen, P., Le Quack, P., . . Nordentoft, M. (2011). Predictors of recovery from psychosis Analyses of clinical and social factors associated with recovery among patients with first-episode psychosis after 5 years. Schizophrenia Research, 125(2-3), 257-266. doi:10.1016/j.schres.2010.10.013

Allen, N. B., Hetrick, S. E., Simmons, J. G., \& Hickie, I. B. (2007). Early intervention for depressive disorders in young people: the opportunity and the (lack of) evidence. Medical Journal of Australia, 187(7 Suppl), S15-S17.

American Psychiatric Association. (2013). Diagnostic and Statistical Manual of Mental Disorders (5th ed.). Arlington, VA: American Psychiatric Publishing.

Andrews, G., \& Wilkinson, D. D. (2002). The prevention of mental disorders in young people. Medical Journal of Australia, 177(7), S97.

Birchwood, M., \& Fiorillo, A. (2000). The critical period for early intervention. Psychiatric Rehabilitation Skills, 4(2), 182-198. doi:10.1080/10973430008408405

Chanen, A. M., Jovev, M., McCutcheon, L. K., Jackson, H. J., \& McGorry, P. D. (2008). Borderline personality disorder in young people and the prospects for prevention and early intervention. Current Psychiatry Reviews, 4(1), 48-57. doi:10.2174/157340008783743820

Chanen, A. M., \& McCutcheon, L. (2013). Prevention and early intervention for borderline personality disorder: current status and recent evidence. British Journal of Psychiatry Supplement, 54, s24-29. doi:10.1192/bjp.bp.112.119180 
Chanen, A. M., McCutcheon, L., \& Kerr, I. B. (2014). HYPE: A Cognitive Analytic Therapy-Based Prevention and Early Intervention Programme for Borderline Personality Disorder. In C. Sharp \& J. L. Tackett (Eds.), Handbook of Borderline Personality Disorder in Children and Adolescents (pp. 361-383). New York, NY: Springer New York.

Cooper, C. L., \& Lu, L. (2016). Presenteeism as a global phenomenon: Unraveling the psychosocial mechanisms from the perspective of social cognitive theory. Cross Cultural \& Strategic Management, 23(2), 216-231. doi:10.1108/CCSM-09-2015-0106

First, M. B., Gibbon, M., Spitzer, R. L., Williams, J. B. W., \& Benjamin, L. S. (1997). User's guide for the structured clinical interview for DSM-IV Axis II personality disorders. . Washington,DC: American Psychiatric Press.

Fraser, R., Berger, G., Killackey, E., \& McGorry, P. (2006). Emerging psychosis in young people - Part 3 - key issues for prolonged recovery. Australian Family Physician, 35(5), 329-333.

Gowers, S., Harrington, R., Whitton, A., Lelliott, P., Beevor, A., Wing, J., \& Jezzard, R. (1999). Brief scale for measuring the outcomes of emotional and behavioural disorders in children. Health of the Nation Outcome Scales for children and Adolescents (HoNOSCA). The British Journal of Psychiatry, 174(5), 413-416. doi:10.1192/bjp.174.5.413

Jackson, H. J., \& McGorry, P. D. (Eds.). (2009). The Recognition and Management of Early Psychosis: A Preventative Approach (Second ed.). New York: Cambridge University Press.

Kessler, R. C., Berglund, P., Demler, O., Jin, R., Merikangas, K. R., \& Walters, E. E. (2005). Lifetime prevalence and age-of-onset distributions of DSM-IV disorders in the National Comorbidity Survey Replication. Archives of general psychiatry, 62(6), 593-602. doi:10.1001/archpsyc.62.6.593

Killackey, E., Jackson, H., Gleeson, J., Hickie, I., \& McGorry, P. (2006). Exciting career opportunity beckons! Early intervention and vocational rehabilitation in first-episode psychosis: employing cautious optimism. Australian and New Zealand Journal of Psychiatry, 40(11-12), 951-962. doi:10.1080/j.1440-1614.2006.01918.x

Killackey, E., Jackson, H. J., \& McGorry, P. D. (2008). Vocational intervention in first-episode psychosis: individual placement and support v. treatment as usual. The British Journal of Psychiatry, 193(2), 114-120. doi:10.1192/bjp.bp.107.043109

Kroenke, K., \& Spitzer, R. L. (2002). The PHQ-9: a new depression diagnostic and severity measure. Psychiatric annals, 32(9), 509-515. doi:10.3928/0048-5713-20020901-06

Leamy, M., Bird, V., Le Boutillier, C., Williams, J., \& Slade, M. (2011). Conceptual framework for personal recovery in mental health: systematic review and narrative synthesis. British Journal of Psychiatry, 199(6), 445-452. doi:10.1192/bjp.bp.110.083733

Major, B. S., Hinton, M. F., Flint, A., Chalmers-Brown, A., McLoughlin, K., \& Johnson, S. (2010). Evidence of the effectiveness of a specialist vocational intervention following first episode psychosis: a naturalistic prospective cohort study. Social Psychiatry and Psychiatric Epidemiology, 45(1), 1-8. doi:10.1007/s00127-009-0034-4

Marwaha, S., \& Johnson, S. (2004). Schizophrenia and employment. Social Psychiatry and Psychiatric Epidemiology, 39(5), 337-349. doi:10.1007/s00127-004-0762-4

McGorry, P. D., Edwards, J., Mihalopoulos, C., Harrigan, S. M., \& Jackson, H. J. (1996). EPPIC: an evolving system of early detection and optimal management. Schizophrenia bulletin, 22(2), 305.

Merry, S. N. (2007). Prevention and early intervention for depression in young people-a practical possibility? Current Opinion in Psychiatry, 20(4), 325-329. doi:10.1097/YCO.0b013e3281bc0d19

Morgan, C., Charalambides, M., Hutchinson, G., \& Murray, R. M. (2010). Migration, ethnicity, and psychosis: toward a sociodevelopmental model. Schizophrenia bulletin, sbq051. doi:10.1093/schbul/sbq051

O’Dea, B., Lee, R. S., McGorry, P. D., Hickie, I. B., Scott, J., Hermens, D. F., . . Pantelis, C. (2016). A prospective cohort study of depression course, functional disability, and NEET status in help- 
seeking young adults. Social Psychiatry and Psychiatric Epidemiology, 51(10), 1395-1404. doi:10.1007/s00127-016-1272-x

Organisation for Economic Co-operation and Development (OECD). (2011). Education at a Glance 2011: OECD Indicators. Retrieved from

Organisation for Economic Co-operation and Development (OECD). (2012). NEET rates among youth in OECD countries: Percentage of population aged 15-24. OECD Employment Outlook 2012. OECD Publishing.

Organisation for Economic Co-operation and Development (OECD). (2015). Mental Health and Work: Australia. Retrieved from Paris:

Organisation for Economic Co-operation and Development (OECD). (2017). Youth unemployment rate (indicator).

Rice, S. M., Halperin, S., Cahill, S., Cranston, I., Phelan, M., Hetrick, S. E., . . Davey, C. G. (2017). The Youth Mood Clinic: an innovative service for the treatment of severe and complex depression. Australasian Psychiatry, 25(2), 112-116.

Sio, I. T., Chanen, A. M., Killackey, E. J., \& Gleeson, J. (2011). The relationship between impulsivity and vocational outcome in outpatient youth with borderline personality features. Early intervention in psychiatry, 5(3), 249-253. doi:10.1111/j.1751-7893.2011.00271.x

Viera, A. J., \& Garrett, J. M. (2005). Understanding interobserver agreement: the kappa statistic. Family Medicine, 37(5), 360-363.

Waghorn, G., Chant, D., Lloyd, C., \& Harris, M. (2011). Earning and learning among Australian community residents with psychiatric disorders. Psychiatry research, 186(1), 109-116. doi:10.1016/j.psychres.2010.07.037

Waghorn, G., \& Lloyd, C. (2005). The employment of people with mental illness. Advances in Mental Health, 4(2), 129-171. doi:10.5172/jamh.4.2.129

Whooley, M. A., Kiefe, C. I., Chesney, M. A., Markovitz, J. H., Matthews, K., \& Hulley, S. B. (2002). Depressive symptoms, unemployment, and loss of income: the CARDIA Study. Archives of Internal Medicine, 162(22), 2614-2620. doi:10.1001/archinte.162.22.2614

Wing, J., Beevor, A., Curtis, R., Park, S., Hadden, S., \& Burns, A. (1998). Health of the Nation Outcome Scales (HoNOS). Research and development. The British Journal of Psychiatry, 172(1), 11-18. doi:10.1192/bjp.172.1.11

Workplace Gender Equality Agency. (2014). Gender workplace statistics at a glance. Retrieved from https://www.wgea.gov.au/sites/default/files/Stats at a glance.pdf

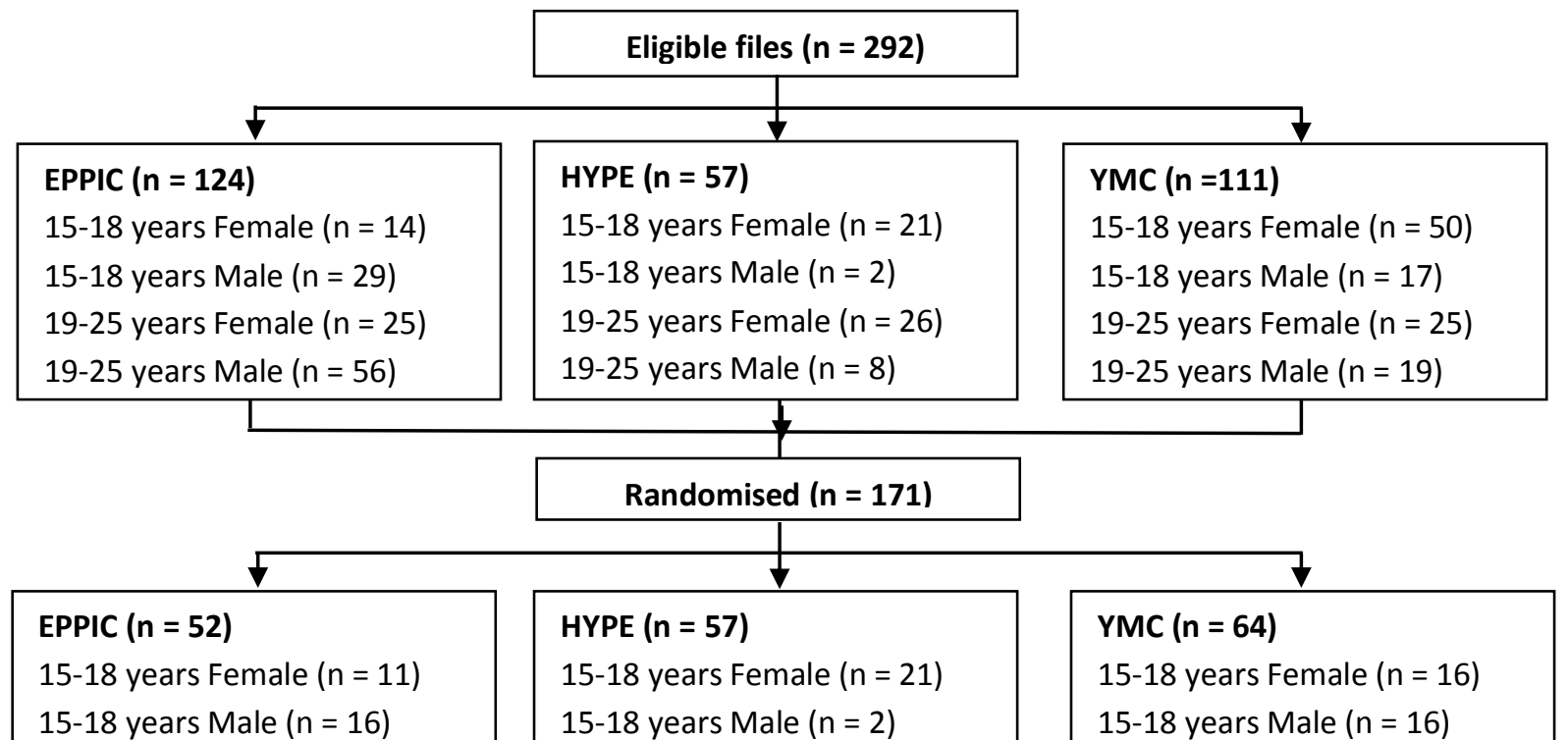


Figure 1. Flow of participant files throughout the study

Note. EPPIC = Emerging Psychosis Prevention and Intervention Centre; HYPE = Helping Young People Early (emerging BPD) clinic; YMC = Youth Mood Clinic

${ }^{a}$ There is a percentage of young people who are registered and assessed with Orygen, but at assessment are deemed ineligible for treatment due to presentation not meeting the required severity

${ }^{\mathrm{b}}$ There were no further files to re-randomise

${ }^{\mathrm{C}} 5$ re-randomised, of these 3 excluded ( 2 transferred between clinics, 1 not eligible), further 2 resampled 

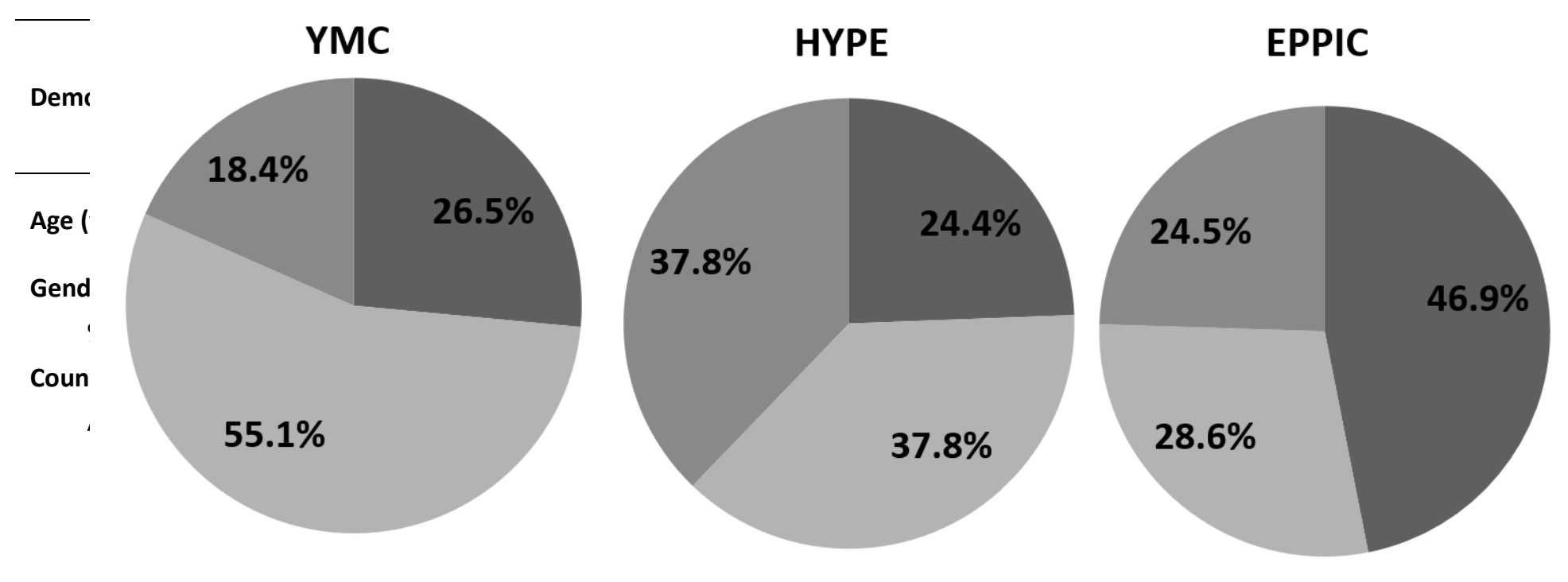

Not in Education, Employment or Training

Partially engaged in employment/education or both

- Fully engaged in employment/education or both

Figure 2. Quality of engagement in employment and/or education.

Note. $\mathrm{YMC}=$ Youth Mood Clinic for severe and complex depression, $n=49 ; \mathrm{HYPE}=$ Helping Young People Early for borderline personality disorder pathology, $n=45$; EPPIC = Early Psychosis Prevention and Intervention Centre, $\mathrm{n}=49$. 


\section{English}

English Proficiency

Speaks English well or very well

Interpreter required

$$
\% \text { Yes }
$$

Indigenous Status

$\%$ Not Aboriginal or Torres Strait Islander

\section{Government Benefit}

$$
\% \text { receiving }
$$

Marital status

Never married

Living status

Alone
Friends
Parents
Defacto/Husband/Wife
Other

Housing

House or flat

MHA Legal status Informal

Hospitalisation at registration

\begin{tabular}{cccc}
$\%(n)$ & $92(46)$ & $100(41)$ & \\
\cline { 3 - 4 } & & & Clin \\
& & Mood & BP \\
& $93.9(46)$ & $100(41)$ & 95.6
\end{tabular}

$89.4(42) \quad 93.5(129)$

BPD

Psychosis

TOTAL5

x294 Sig.

2.57

.277

$\%(n) \quad 4.3(2) \quad 0(0)$

7 (3)

$4(5)$

$\%(n) \quad 100(48)$

$95.3(41)$

100 (46)

98.5 (135)

4.44

.109

1.43

.489

$\%(n) \quad 15(6)$

23.1 (9)

$26.7(12)$

21.8 (27)

$\%(n) \quad 93.5(43)$

$88.9(40)$

95.9 (47)

92.9 (130)

1.79

.409

6.36

\begin{tabular}{|c|c|}
\hline$\%(n)$ & $0(0)$ \\
\hline$\%(n)$ & $18(9)$ \\
\hline (n) & 68 \\
\hline (n & $4(2)$ \\
\hline $\ln$ & 10 \\
\hline
\end{tabular}

.607

4.5 (2)

4.1 (2)

2.8 (4)

$13.6(6)$

$12.2(6)$

14.7 (21)

54.5 (24)

$67.3(33)$

$63.6(91)$

11.4 (5)

$6.1(3)$

7 (10)

$15.9(7)$

$10.2(5)$

11.9 (17)

2.97

.226

$\%(n) \quad 95.9(47)$

95.5 (42)

88 (44)

93 (133)

$\%(n) \quad 97.7(42) \quad 100(41)$

$66.7(28)$

88.1 (111)
$27.69<.001 * * *$

$7.15 \quad .028 *$

Table 1. Demographic characteristics of participants at service entry, according to clinic attended Note. $\mathrm{M}=$ mean; $\mathrm{SD}=$ standard deviation; $\mathrm{BPD}=$ borderline personality disorder; Sig. = significance; $\mathrm{MHA}=$ Mental Health Act. MHA Legal status is whether a young person is informal (voluntarily receiving treatment) or formal (involuntary patient under legislation). The denominator for percentages may vary according to missing data, therefore denominator does not always equal group total (Mood=50; BPD=45; Psychosis $=50 ;$ TOTAL=145). ${ }^{*} p<.05 .{ }^{* *} p<.01 .{ }^{* * *} p<.001$. 


\section{Education Variables}

Current Education Status

$4.15 \quad .386$

Not studying

Partial engagement in study

Studying

Highest Education Completed

Secondary Yr 7-10

Secondary Yr 11-12

Tertiary commenced

Tertiary completed

HONOSCA Item 13: Poor School

\section{Attendance}

No to minimal problem

Moderate to severe problem

$\begin{array}{ll}\%(n) & 44.7(21) \\ \%(n) & 42.6(20) \\ \%(n) & 12.8(6)\end{array}$

41.5 (17)

34.1 (14)

$24.4(10)$

55.8 (24)

47.3 (62)

$\%(n)$

$12.8(6)$

27.9 (12)

35.1 (46)

$16.3(7)$

$17.6(23)$

7.05

.317

$\begin{array}{cc}\%(n) & 35.4(17) \\ \%(n) & 35.4(17) \\ \%(n) & 22.9(11) \\ \%(n) & 6.3(3)\end{array}$

57.1 (24)

35.4 (17)

42.0 (58)

$31.0(13)$

39.6 (19)

35.5 (49)

9.5 (4)

20.8 (10)

$18.1(25)$

$2.4(1)$

$4.2(2)$

$4.3(6)$

Table 2. Univariate analyses comparing groups on current educational status, highest education completed and HONOSCA Item 13: poor school attendance

Note. HONOSCA $=$ Health of the Nation Outcome Scale Child and Adolescent. Reduced $n$ as HONOSCA only completed for those under 18 years; BPD = borderline personality disorder; Sig. = significance. The denominator for percentages may vary according to missing data, therefore denominator does not always equal group total (Mood=50; BPD=45; Psychosis=50; TOTAL=145). ${ }^{*} p<.05 .{ }^{* *} p<.01$. $* * * p<.001$. 
Table 3. Univariate analyses comparing groups on current employment status and HONOS Item 12: problems with occupation and activities

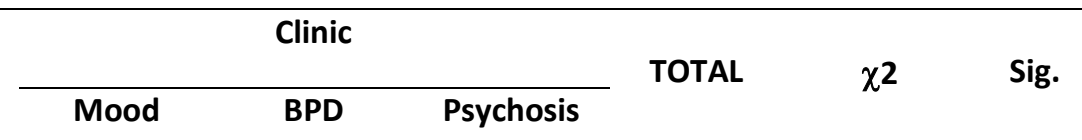

Employment Variables

\begin{tabular}{|c|c|c|c|c|c|c|c|}
\hline Employment Status & & & & & & 2.62 & .624 \\
\hline Unemployed & $\%(n)$ & $50.0(17)$ & $46.9(15)$ & $62.9(22)$ & $53.5(54)$ & & \\
\hline $\begin{array}{l}\text { Partial engagement in } \\
\text { employment }\end{array}$ & $\%(n)$ & $8.8(3)$ & $12.5(4)$ & $11.4(4)$ & $10.9(11)$ & & \\
\hline Employed & $\%(n)$ & $41.2(14)$ & $40.6(13)$ & $25.7(9)$ & $35.6(36)$ & & \\
\hline $\begin{array}{l}\text { HONOS Item 12: Problems with } \\
\text { occupation and activities }\end{array}$ & & & & & & 0.96 & .619 \\
\hline No to minimal problem & $\%(n)$ & $53.6(15)$ & $48.3(14)$ & $60.6(20)$ & $54.4(49)$ & & \\
\hline Moderate to severe problem & $\%(n)$ & $46.4(13)$ & $51.7(15)$ & $39.4(13)$ & $45.6(41)$ & & \\
\hline
\end{tabular}

Note. HONOS= Health of the Nation Outcome Scale. Reduced $n$ as HONOS only completed for those 18 years and over; BPD = borderline personality disorder; Sig. = significance. The denominator for percentages may vary according to missing data, therefore denominator does not always equal group total (Mood=50; BPD=45; Psychosis=50; TOTAL=145). ${ }^{*} p<.05 .{ }^{* * p}<.01 .{ }^{* * *} p<.001$. 
Table 4. Univariate analyses comparing groups on level of engagement and type of vocational activity

\begin{tabular}{|c|c|c|c|c|c|c|c|}
\hline & & \multicolumn{3}{|c|}{ Clinic } & \multirow{3}{*}{ TOTAL } & \multirow{3}{*}{$x^{2}$} & \multirow{3}{*}{ Sig. } \\
\hline & & Mood & BPD & Psychosis & & & \\
\hline \multicolumn{5}{|l|}{ Overall Engagement Variables } & & & \\
\hline Quality of Engagement & & & & & & 12.35 & $.015^{*}$ \\
\hline NEET & $\%(n)$ & $26.5(13)$ & $24.4(11)$ & $46.9(23)$ & $32.9(47)$ & & \\
\hline Partially vocationally engaged & $\%(n)$ & $55.1(27)$ & $37.8(17)$ & $28.6(14)$ & $40.6(58)$ & & \\
\hline Fully vocationally engaged & $\%(n)$ & $18.4(9)$ & $37.8(17)$ & $24.5(12)$ & $26.6(38)$ & & \\
\hline Type of Vocational Activity & & & & & & 8.48 & .205 \\
\hline NEET & $\%(n)$ & $26.5(13)$ & $24.4(11)$ & $46.9(23)$ & $32.9(47)$ & & \\
\hline Engaged only in education & $\%(n)$ & $40.8(20)$ & $37.8(17)$ & $26.5(13)$ & $35.0(50)$ & & \\
\hline Engaged only in employment & $\%(n)$ & $14.3(7)$ & $22.2(10)$ & $10.2(5)$ & $15.4(22)$ & & \\
\hline $\begin{array}{l}\text { Engaged in both education and } \\
\text { employment }\end{array}$ & $\%(n)$ & $18.4(9)$ & $15.6(7)$ & $16.3(8)$ & $16.8(24)$ & & \\
\hline
\end{tabular}

Note. $\mathrm{BPD}=$ borderline personality disorder; Sig. = significance; NEET= not in education, employment or training. The denominator for percentages may vary according to missing data, therefore denominator does not always equal group total (Mood=50; BPD=45; Psychosis=50; TOTAL=145). ${ }^{*} p<.05$. 


\section{University Library}

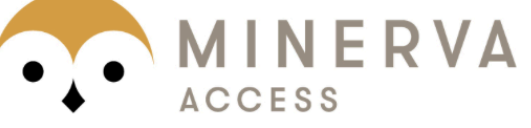

A gateway to Melbourne's research publications

Minerva Access is the Institutional Repository of The University of Melbourne

Author/s:

Caruana, E;Cotton, SM;Farhall, J;Parrish, EM;Chanen, A;Davey, CG;Killackey, E;Allott, K

Title:

A Comparison of Vocational Engagement Among Young People with Psychosis, Depression and Borderline Personality Pathology

Date:

2018-08-01

Citation:

Caruana, E., Cotton, S. M., Farhall, J., Parrish, E. M., Chanen, A., Davey, C. G., Killackey, E. \& Allott, K. (2018). A Comparison of Vocational Engagement Among Young People with Psychosis, Depression and Borderline Personality Pathology. COMMUNITY MENTAL HEALTH JOURNAL, 54 (6), pp.831-841. https://doi.org/10.1007/s10597-017-0197-5.

Persistent Link:

http://hdl.handle.net/11343/283318 